\title{
Genotype-Environment Interaction in ADHD: Genetic Predisposition Determines the Extent to Which Environmental Influences Explain Variability in the Symptom Dimensions Hyperactivity and Inattention
}

\author{
Inga Schwabe*1, Miljan Jović ${ }^{2}$, Kaili Rimfeld ${ }^{3}$, Andrea G. Allegrini ${ }^{3}$ and \\ Stéphanie M. van den Berg ${ }^{2}$
}

1 Department of Methodology and Statistics, Tilburg University, the Netherlands

2 Department of Research Methodology, Measurement and Data Analysis (OMD), University of Twente, the Netherlands

3 Social, Genetic and Developmental Psychiatry Centre, Institute of Psychiatry, Psychology and Neuroscience, King's College London, UK

*Towhom correspondence should be addressed.Email:I.Schwabe@uvt.nl.

Contact information corresponding author:

PO Box 90153, 5000 LE Tilburg, the Netherlands, I.Schwabe@uvt.nl

\begin{abstract}
Although earlier studies in the field of behaviour genetics have shown that individual differences on the spectrum of the attention deficit hyperactivity disorder (ADHD) are highly heritable, emerging evidence suggests that symptoms are associated with complex interactions between genes and environmental influences. This study investigated whether a genetic predisposition for the symptom dimensions hyperactivity and inattention determines the extent to which uniqueenvironmental influences explain variability in these symptoms. To this purpose, we analysed item-level scores of 2168 twin pairs who completed both the Strengths and Difficulties Questionnaire (SDQ) and the Strength and Weaknesses of ADHD Symptoms and Normal Behavior (SWAN) questionnaire. To maximize the psychometric information to measure ADHD symptoms, psychometric analyses were performed to investigate whether the items from the two questionnaires can be combined to form two longer subscales. In the estimation of genotype-
\end{abstract}


environment interaction, we corrected for error variance heterogeneity in the measurement of ADHD symptoms through the application of item response theory (IRT) measurement models. Results indicated that unique-environmental influences were more important in creating individual differences in both hyperactivity and inattention for twins with a genetic predisposition for these symptoms than for twins without such a predisposition.

Keywords: ADHD, Hyperactivity, Inattention, Genotype-environment interaction, IRT, Twin study, Measurement error, Environmental influences

\section{Introduction}

Attention deficit hyperactivity disorder (ADHD) affects roughly $5 \%$ of the worldwide child and adolescent (6 to 12 years) population (Polanczyk, De Lima, Horta, Biederman \& Rohde, 2007). Symptoms of ADHD are measured on two different core dimensions, hyperactivity and inattention, which form the basis of the current Diagnostic and Statistical Manual of Mental Disorders subtype classification system. Depending on the (combination of) symptoms, individuals who suffer from ADHD are easily distracted during tasks and conversations, experience severe difficulty to sustain activities or show deficit in inhibitory control (Scheres et al., 2003). This can cause difficulties with school education, lead to emotional and behavioural difficulties or impair peer relationships. For 50\% of the ADHD patient population, symptoms persist into adulthood (Schmitz, Polanczyk \& Rohde, 2007) and may have a significant impact on academic and personal life, due to for example poorer academic development, family problems or higher health care expenses (Coghill et al., 2008).

With heritability estimates ranging from 70 to $90 \%$ for both symptom dimensions, the results of genetically-informative twin studies have consistently demonstrated that variation in the two 
core dimensions of ADHD is strongly influenced by genetic factors (e.g., Willcutt, 2005; Rietveld, Hudziak, Bartels, van Beijsterveldt \& Boomsma, 2004; Hudziak, Rudiger, Neale, Heath \& Todd, 2000; Faraone \& Doyle, 2002). A comprehensive meta-analysis by Nikolas \& Burt (2010) that was based on the results of 79 twin and adoption studies showed that, while both dimensions are highly heritable, hyperactivity and inattention are distinct as to the amount of additive and dominant genetic influences that are important in creating individual differences. Genetic influences can either be additive or non-additive (dominant): While additive genetic effects represent the summed effect of genetic influences across multiple loci, dominant genetic influences are multiplicative effects that represent interactions among alleles. Nikolas \& Burt (2010) showed that variance within the hyperactivity dimension could be mainly explained by additive genetic influences (71\%) while dominant genetic influences contributed only a small proportion to the variance $(2 \%)$. For attention, additive genetic influences were also the most important source of individual differences, but the amount was smaller compared to hyperactivity (56\%) while the contribution of dominant genetic influences was larger (15\%). Furthermore, the remaining variance (27\% for hyperactivity and $29 \%$ for inattention) could be mostly explained by environmental influences that are unique to a single twin (i.e., uniqueenvironmental influences). That is, the shared environment (e.g., environmental factors common to twins from the same family, such as their home environment) did not account for a significant proportion of individual differences, a result replicated in many other studies. The authors of the meta-analysis furthermore found that results were consistent among several moderating factors, including gender, age and measurement instrument.

However, while genetic influences are strongly implicated in the aetiology of ADHD, it remains difficult to characterize the particular environmental circumstances under which ADHD 
symptoms emerge. The reason for this difficulty might lie in the complex nature of the disease: Similar to other mental disorders, ADHD likely results from the combined action of multiple genes with small effects and various environmental risk factors. Additionally, a simple distinction into nature on the other hand and nurture on the other hand might be too simplistic, because there is emerging evidence that complex traits are associated with complex interactions between genetic- and environmental influences. In this manuscript, we focus on the latter: How do genes and environmental stressors interact to create individual differences on the ADHD spectrum?

\section{Genotype-environment interaction}

A genotype-environment interaction formally refers to the situation in which the relative importance of environmental influences in explaining individual differences in a trait is conditional on the genotype. Applied to the mental disease ADHD, this could for example mean that environmental influences are more important in creating individual differences in children with a genetic predisposition towards ADHD than for children without such a predisposition. Various studies suggest that genotype-environment interaction is an important phenomenon in mental disorders (see e.g., Wermter al., 2010). Yet, research on genotype-environment interaction has not been a prominent focus in ADHD research. In one of the, to our knowledge, few published papers that examine genotype-environment interaction in ADHD in twin data, Gould, Coventry, Olson and Byrne (2018) investigated whether there is an interaction between additive genetic effects on ADHD symptoms and two specific shared environmental factors: socioeconomic status (SES) and chaos (household disorganization). Based on a population sample of 520 twin pairs, results showed that neither SES nor chaos were associated with a change in the extent to which genetic influences explain variability in ADHD symptoms. Note 
however that a plausible source for these non-findings might be insufficient power - earlier research has shown that much larger sample sizes are needed to detect genotype-environment interaction when using the method that was used by Gould et al. (2018) (Hanscombe et al., 2012).

Gould et al. (2018) tested genotype-environment interaction in the case that environmental influences are specific, measured variables. They tested two variables, but there are hundreds of possible environmental factors that could interact with additive genetic effects. Instead of testing them all separately, in the present study we applied an omnibus test to assess whether there is any statistically significant interaction between genetic influences and unique-environmental influences. Thus, instead of focusing on one (or more) measured environmental stressors, this method parametrizes environmental influences as one (unobserved) latent variable and we then test if this latent variable interacts with genetic effects. As a result, we focus on the whole range of possible (relevant) environmental factors (summarized in one latent variable), resulting in much larger power compared to focusing on one or more specific environmental stressors(for technical details, see Schwabe \& van den Berg, 2014). This results in proper statistical power to determine - in an exploratory way - whether there is any genotype-environment interaction at all. If indeed an interaction effect is found, future research on the aetiology of ADHD can focus on the exact nature of this effect by collecting specific unique-environmental measures. Unlike Gould et al. (2018), we focus specifically on unique-environmental influences (factors not shared by a twin pair), since earlier results showed that shared environmental influences contribute no variance in $\mathrm{ADHD}$, making it unlikely to find an interaction between genetic effects and shared environmental factors. 


\section{Psychometric issues in the diagnosis of ADHD symptoms}

Measurement of ADHD based on questionnaire data typically involves the construction of a composite score such as summation of endorsed symptoms or adding up a twin's individual answers to all questionnaire items to form a sum-score. However, applying this approach comes with a number of disadvantages in terms of methodological consequences and clinical interpretation. By calculating a composite score, all individual symptoms are weighted equally. Consequently, if one symptom is more indicative of the underlying dimension of ADHD than other symptoms, this is ignored. Furthermore, the use of a composite scores neglects the uncertainty (e.g., the measurement error) that results from using only a limited set of items. This leads to a confounded measure of the underlying latent (i.e., not directly observable) trait, which can result not only in biased heritability estimates (van den Berg, Glas \& Boomsma, 2007), but also in the spurious finding of a genotype-environment interaction effect. The latter has to do with the fact that the amount of information obtained from a questionnaire typically varies for different levels of the measured trait (see e.g. Loehlin and Nichols, 1976 and Waldron, 2000). For example, while existing IQ tests usually show little measurement error for average students, scale scores can be very unreliable for high performing students because there is only little information provided by only a few very difficult items. This is also the case for clinical scales: If both affected and healthy individuals that suffer from ADHD are assessed with a scale that contains many extreme items (for example: "I always have a hard time to pay attention"), then scale scores may be very reliable for twins that show ADHD symptoms, but very unreliable for healthy twins. In extreme situations (such as the ones just discussed), this often leads to floor or ceiling effects. A floor (ceiling) effect represents smaller individual differences at the lower (higher) end of the measurement scale. With other words: Measurement error is not 
homogeneous. This heterogeneity in measurement error leads to a skewed distribution of composite scores (e.g., sum scores). Research shows that this statistical artefact can result in the finding of a spurious genotype-environment interaction effect. For example, when our sample consists of high-school students and the conducted mathematics test is too easy for the most able twins, this will result in smaller score differences within highly able twin pairs than within average or less able twins (a ceiling effect). As a result, twins with a higher sum score seem more alike. In case of a clinical scale, most healthy twins might not endorse extreme items which will result in smaller score differences within health twin pairs than within moderately ill or very ill twins (a floor effect). As a result, twins with a lower sum score seem more alike. In both scenarios, the finding of a spurious GxE effect can be expected - a positive effect in case of a ceiling effect and a negative effect in case of the floor effect (see e.g. Eaves, Last, Martin \& Jinks, 1977; van der Sluis, Dolan, Neale, Boomsma \& Posthuma, 2006; Schwabe \& van den Berg, 2014; Molenaar \& Dolan, 2014).

Using simulation studies, Schwabe \& van den Berg (2014; see also Molenaar \& Dolan, 2014) showed that this bias can be prevented by analysing raw item scores, which can be done by simultaneously estimating the genetic twin model with an item response theory (IRT) model. Instead of ignoring measurement error as is done when using a composite score (like a sum score), the genetic variance decomposition (including genotype-environment interaction) is done directly on the latent variable that is corrected for measurement error. Crucial to this approach is that the analysis takes place in one unified model (estimating IRT and genetic model at the same time). When a so-called two-step procedure (i.e., using a measurement model to estimate latent trait scores and using these scores in a genetic design in a second step), the uncertainty of the measurement of the trait is not fully taken into account and we can still expect spurious GxE. 


\section{This research}

We explored genotype-environment interaction in 2168 16-year-old twins who completed both the Strengths and Difficulties Questionnaire (SDQ, Goodman, 1997) and the Strength and Weaknesses of ADHD Symptoms and Normal Behavior (SWAN) questionnaire (Swanson et al., 2005). To maximize the psychometric information available to measure ADHD symptoms, psychometric analyses were performed first to investigate whether the items from the respective subscales can be combined to form one more reliable scale. Next, genotype-environment interaction was studied separately for the two dimensions hyperactivity and inattention applying the methodology from Schwabe \& van den Berg (2014). This method corrects for error variance heterogeneity in the measurement of the ADHD symptoms. As a result, our results regarding genotype-environment interaction as well as heritability estimates are free of any statistical artefacts due to measurement error.

\section{Method}

\section{Sample}

The data originate from the Twins Early Development Study (TEDS), which is a longitudinal twin study that recruited over 16,000 twin-pairs born between 1994 and 1996 in England and Wales through national birth records. From the total sample that was available to us, $N=11690$ individual twins, $N=656$ individual twins were excluded from the analysis. $N=340$ individual twins were excluded due to medical reasons, $N=168$ because they were classified prenatal outliers, $N=64$ due to unknown sex or zygosity, $N=28$ due to absence of first contact data and for $N=56$ multiple of the mentioned exclusion criteria applied (i.e., for $N=32$ individual twins first contact data was absent and sex or zygosity was unknown, $N=22$ were classified as both 
medical reason exclusion as prenatal outlier and $N=2$ were excluded due do medical reasons and unknown sex or zygosity).

All twin pairs excluded from analysis were from the same twin families (e.g., both first and cotwin had to be excluded), leading to a total $N$ of 5517 twin pairs ( $\mathrm{N}=11034$ individual twins), of which 1991 monozygotic (MZ) twin pairs and 3526 dizygotic (DZ) twin pairs. Of the MZ pairs, 858 twin pairs were male and 1133 female. Of the DZ twin pairs, 796 pairs were male, 994 pairs were female and 1736 were opposite sex twin pairs. Mean age of the twins at the time of return of the questionnaires was 16 years and three months $(\mathrm{SD}=0.75$, minimum age $=14$ years and 9 months and maximum age $=18$ years and 7 months $)$. For the majority of the twins $(N=5293$ twin pairs), English was the first language spoken at home and their ethnic origin was white ( $N=$ 5142 twin pairs).

To avoid bias in the psychometric analyses, due to the dependency in the data (e.g., twins being nested within families), we randomly selected one twin from every family for psychometric analyses, which reduced the dataset to a sample size of $N=5517$ individual twins. Of these individual twins, only the data of twins who had answered both questionnaires completely (e.g., had no missing data on the combined scales) were used for psychometric analyses. This reduced the dataset to $N=1043$ individual twins for the combined hyperactivity scale and to $N=1037$ individual twins for the combined inattention scale.

Based on the results of the psychometric analyses, it was decided which subset of the data was used for genetic analyses (either only the items originating from the longer SWAN subscale or the combined scale based on both items from the SWAN and SDQ). Of this subset, data of families with missing data on all items were excluded from the analysis. This resulted in a sample size of $N=1084$ twin pairs (415 MZ twin pairs and $669 \mathrm{DZ}$ twin pairs) for both 
hyperactivity and inattention scale. Out of the remaining twin families, $N=1033(95 \%)$ twin families had no missing data on the hyperactivity scale, within $N=20(2 \%)$ families a single item answer was missing, $N=8(1 \%)$ families missed 8 or fewer item scores and $N=23(2 \%)$ had 9 unknown item scores. Concerning the inattention scale data, for $N=1016(94 \%)$ twin pairs all item data were complete, within $N=34(3 \%)$ families a single item answer was unknown, $N$ $=11(1 \%)$ twin pairs were missing 2 or up to 7 item scores and $N=23(2 \%)$ families were missing $9(N=22$ families $)$ or $10(N=1$ twin family) item scores.

\section{ADHD scales}

The Strengths and Difficulties Questionnaire (SDQ) is a brief questionnaire for children and young people, meant for emotional and behavioural screening. With a total of 25 items, the hyperactivity/impulsive subscale of the questionnaire consists of 5 items and uses a three-point scale $(0-$ not true, 1 - quite true and 2 - very true $)$. Three of the items of this subscale measure inattention and two items hyperactivity. The items of the SDQ were part of the test booklet that was sent to twins at the age of 16 and answered by themselves (not their parents or teachers).

Developed by Swanson et al. (2006), the Strength and Weaknesses of ADHD symptoms and Normal Behavior (SWAN) questionnaire consists of two subscales of each 9 items, derived from the DSM-IV-TR (American Psychiatric Association, 2008). The two subscales measure inattention and hyperactivity respectively using a seven-point scale of behaviour $(1-$ far below average, 2 - below average, 3 - slightly below average, 4 - average, 5 - slightly above average, 6 - above average and 7 - far above average). Like the items from the SDQ, the items of the SWAN were part of the test booklet that was sent to twins at the age of 16 and answered by 
themselves (not their parents or teachers). The appendix contains a list with all items included in the hyperactivity and inattention subscales of the SWAN and SDQ.

\section{Psychometric analyses}

Psychometric analyses were conducted separately for hyperactivity (e.g., 9 items from the SWAN questionnaire and 2 items from the SDQ questionnaire) and for inattention (e.g., 9 items from the SWAN questionnaire and 3 items from the SDQ questionnaire). To investigate whether the two questionnaires measure the same underlying trait, we formed two long scales consisting of a total of 11 items (hyperactivity) and 12 items (inattention) respectively. The item scores of the two combined scales were recoded such that a higher score on the combined scale reflected a higher trait score (i.e., a high degree of hyperactivity and inattention respectively).

To test whether combining the items from the different scales resulted in one unidimensional scale that measures the same latent trait (hyperactivity and inattention, respectively), multiple IRT models were estimated (e.g., the partial credit model (PCM), the generalized partial credit model (GPCM) and the graded response model (GRM)). Based on the lowest Akaike Information Criterion (AIC), the best fitting IRT model was selected and used to investigate item information curves and item fit of the combined scales. For the estimation of all models, the R package mirt (Chalmers, 2012) was used.

\section{Genetic models}

Employing twin data allows to fit different genetic models. The most commonly used model is the so-called ACE model. The ACE model decomposes the total observed variance in the trait, $\sigma_{P}^{2}$, into variance that can be explained by additive genetic influences (denoted as $\sigma_{A}^{2}$ ), common-

environmental influences (represented by $\sigma_{C}^{2}$ ) and unique-environmental influences (denoted by $\sigma_{E}^{2}$ ). Common-environmental influences (C) are parametrized to be perfectly correlated within 
the same family (one twin pair) and represent influences that lead to resemblance in twins that cannot be attributed to their genetic resemblance, such as the family environment or other shared environmental influences (e.g., the same friends or teachers). Unique-environmental influences (E, also referred to as non-shared environmental influences) are parametrized to be correlated zero within one family (one twin pair) and interpreted as influences that are unique to an individual twin (e.g., the environment that is not shared). In the case of a composite score (e.g., a sum score) that is not adjusted for measurement error, the variance component also contains measurement error. Note that, here, by using the method developed by Schwabe \& van den Berg (2014), we are correcting for the part that contains measurement error (see below for more detail).

It is also possible to fit an AE model in which we set $\mathrm{C}$ to zero or to fit an ADE model in which the D represents dominance effects (e.g., non-additive genetic variance) and the total observed variance is decomposed into variance due to additive-genetic influences $\left(\sigma_{A}^{2}\right)$, variance explained by dominance effects $\left(\sigma_{D}^{2}\right)$ and variance due to unique-environmental influences $\left(\sigma_{E}^{2}\right)$. Note that common-environmental influences and dominant effects cannot be estimated at the same time (within the same model), because this would mean that the model is not identified anymore.

\section{Genotype-environment interaction}

In case of genotype-environment interaction, the amount of variance due to uniqueenvironmental influences is not the same for every twin, but depends on an individual's genotypic value, which is parametrized as a latent (e.g., unobserved) variable. This means that the unique-environmental variance component (e.g., $\left.\sigma_{E}^{2}\right)$ can be larger at either higher or lower 
levels of the genotypic value. For this particular application, this means that uniqueenvironmental influences are either more important for twins with a high genetic predisposition for ADHD (i.e., positive genotype-environment interaction) or for twins with no or only a week genetic predisposition (i.e., negative genotype-environment interaction). A positive genotypeenvironment interaction predicts that twins with the same high genotypic value (genetically predisposed for ADHD) are less similar (more variance) than twins with the same low genotypic value (low predisposition for ADHD). A negative genotype-interaction predicts the opposite.

To model genotype-environment interaction under the ACE and AE model, we portioned variance due to unique-environmental influences into an intercept (representing environmental variance when $A=0$ ) and a slope parameter that is a function of the additive genotypic value $A$ and represents the interaction effect (henceforth referred to as AxE). This makes the uniqueenvironmental influence different for every individual $j$ with additive genotypic value $A_{j}$. To force the variance to be positive, the exponent was taken (cf. SanCristobal-Gaudy et al., 1998).

$$
\sigma_{E j}^{2}=\exp \left(\beta_{0}+\beta_{1} A_{j}\right)
$$

where $\beta_{0}$ denotes the intercept and $\beta_{1}$ is a slope parameter that represents AxE.

Note that the sign of the slope (e.g., $\beta_{1}$ ) determines the direction of the interaction effect and is modelled here as a $(\log )$ linear effect. This means that AxE is interpreted as a linear effect (on the $\log$ scale), assuming that environmental variance is larger at either a higher (positive) or a lower (negative) level of the genotypic value (i.e., larger differences among individuals with similar $A$ ).

Under the ADE model, the AxE interaction effect (in case of the ADE model referred to as GxE) was conditioned on the complete genotype (e.g., both additive genetic and dominant 
genetic effects) instead of only additive genetic effects. This makes variance due to uniqueenvironmental influences different for every individual $j$ with genotypic value $G_{j}$ :

$$
\sigma_{E j}^{2}=\exp \left(\beta_{0}+\beta_{1} G_{j}\right)
$$

where $G_{j}$ represents the genotypic value (including both additive and non-additive genetic effects) of individual $j, \beta_{0}$ denotes the intercept (when $G_{j}=0$ ) and $\beta_{1}$ is a slope parameter that represents GxE.

\section{Measurement model}

Simultaneously to every fitted genetic model, a measurement model in the form of an IRT model was estimated. In IRT models, item scores depend not only on a person's latent trait (in this case, hyperactivity and inattention respectively), but also on the properties of the items that are contained in the questionnaire such as the difficulty of an item. We used the generalized partial credit model (GPCM), which is an IRT model that is suitable for polytomous, ordinal data (Muraki, 1992). The GPCM treats polytomous responses as ordered trait levels and models the probability of endorsing response category $k$ over $k-1$ for item $i$ using both the latent trait value and item parameters. For example, if symptom occurrence is assessed on a scale with 4 answer categories (e.g., "1 - never", "2 - sometimes", 3 - often and 4 - always), we have $K=4$ and a respondent who selects "sometimes" as the response that describes best their situation, is considered to have chosen "sometimes" over "never" and "often" over "sometimes", but to not have chosen "always" over "often". This can be interpreted intuitively as if a respondent "passes" all of the preceding ordered response categories before stopping at the final response which reflects most accurately the person's standing on the latent variable continuum. For each successive response category, the probability of endorsing response category $k$ over $k-1$ for 
item $i$ is assumed to follow a conditional probability that is dependent on an individual's latent trait, the discrimination parameter $\alpha_{i}$ of that item and thresholds for every item category $k$ of item $i, \beta_{i k}$, that a person has to step through in order to reach the next response category. The discrimination parameter represents how well an item discriminates between the various levels of the latent trait (comparable to a factor-loading in structural equation modelling). Note that by adopting the IRT approach we define ADHD as a latent trait that varies on a continuum, rather than a category of a mental disorder.

To identify the IRT model, we set the mean of the latent trait as well as the first threshold for all items to zero (i.e., $\mu=0$ and $\beta_{i 1}=0$ ).

\section{One-step estimation of genetic and measurement model}

Van den Berg et al. (2007) showed that, in order to take full advantage of the IRT approach, the genetic model and the IRT model have to be fitted concurrently. Only then, the uncertainty of the measurement of the trait is fully taken into account and the spurious finding of a genotypeenvironment interaction effect due to heterogeneous measurement error can be prevented. Here we use a Bayesian approach to estimate the parameters from the IRT measurement model and the genetic model at the same time, in a unified model. In Bayesian statistical modelling, inference is based on the joint posterior density of all model parameters, which is proportional to the product of a prior probability and the likelihood function of the observed data (Box \& Tiao, 1992). To obtain this joint posterior density, we applied the Markov chain Monte Carlo (MCMC) algorithm Gibbs sampling (Geman \& Geman, 1984; Gelfand \& Smith, 1990). For a detailed description of the specification of the used genetic models in this context, the reader is referred to van den Berg, Beem \& Boomsma (2006), Schwabe \& van den Berg (2014), Schwabe, Jonker \& van den Berg (2015), Schwabe, Boomsma \& van den Berg (2017) and Schwabe (2017)). 
To determine which genetic model fitted the data best, while, at the same time, being parsimonious, we fitted all possible combinations of genetic models, with and without genotypeenvironment interaction (e.g., AE, AE with AxE, ACE, ACE with AxE, ADE and ADE with GxE) and calculated the deviance information criterion (DIC, Spiegelhalter, Best, Carlin \& van der Linde, 2002). The DIC is a measure that estimates the amount of information that is lost when a given model is used to present the data generating process. It takes into account both goodness of fit and complexity of the model. All estimated genetic models were fitted simultaneously with a measurement model (IRT model) as described above. For the MCMC estimation, we used the freely obtainable MCMC software package JAGS (Plummer, 2003). For further data handling, the statistical programming language $\mathrm{R}$ was used (R Development Core Team, 2008). As an interface from R to JAGS, we used the rjags package (Plummer, 2013).

After a burn-in phase of 25,000 iterations for each separate Markov chain, the characterization of the posterior distribution was based on a total of 30,000 iterations from two separate Markov chains. After inspection of convergence plots, it was decided to increase the burn-in period for the ACE with AxE and AE with AxE models for the inattention subscale data to a total of 45,000 iterations. Even with a large amount of burn-iterations (i.e., 75,000 ), the two MCMC chains of both, the ADE and ADE with GxE model, did not achieve stationarity (i.e., did not approach the joint posterior or target distribution sufficient closely). Convergence plots of all estimated models are not displayed here to save space, but can be obtained from the first author. Note that by applying the IRT approach, we were able to analyze the data on item-level such that the raw data could be used as input in JAGS without having to impute any missing item scores first. JAGS automatically imputes the missing data based on their posterior distribution at every iteration of the algorithm. 
For the genetic model that fitted the data best, we calculated the mean and standard deviation for each parameter as well as the $95 \%$ highest posterior density (HPD, see e.g. Box \& Tiao, 1992) interval. The HPD interval can be interpreted as the Bayesian version of a confidence interval (CI) in frequentist statistics. The influence of a model parameter can be regarded significant when the respective HPD interval does not contain zero (with the exception of the variance components, since these are bounded at zero). Furthermore, in the case that a model that included an interaction effect was chosen as the best fitting model for the data at hand, we also calculated the effect size of the interaction effect. The effect size was defined as the factor with which the environmental variance component increases for an individual with an additive genetic effect of $A_{i}=\sigma_{a}$. For technical details and power calculations, we refer the reader to Schwabe \& van den Berg (2014).

\section{Results}

\section{Psychometric results}

\section{Hyperactivity}

Based on a comparison of different IRT models (e.g., partial credit model (PCM), generalized partial credit model (GPCM) and graded response model (GRM)), the GPCM was selected as the best fitting model for the data at hand and used to investigate item information curves and item fit.

The item information curves that resulted from applying the GPCM to the items of the combined scale (e.g., 9 items from the SWAN questionnaire and 2 items from the SDQ questionnaire) can be found in Figure 1. The item information curves show the amount of information provided by the items taken from the two different questionnaires (SDQ items are 
displayed with a dotted line in the figure). The $\mathrm{Y}$-axis of the figure depicts the item information and the $\mathrm{X}$-axis shows the theta values where a higher value equals a higher degree of inattention. It can be seen that, overall, the combined scale contains more information on the right side of the trait continuum (e.g., above average to a high degree of hyperactivity). Furthermore, generally, items taken from the SWAN questionnaire are more informative. Lastly, whereas the SWAN questionnaire items contain more information on the extremes of the trait continuum (particularly on twins with a high degree of hyperactivity), items that originate in the SDQ questionnaire discriminate mostly among twins with an average trait value (average hyperactivity).

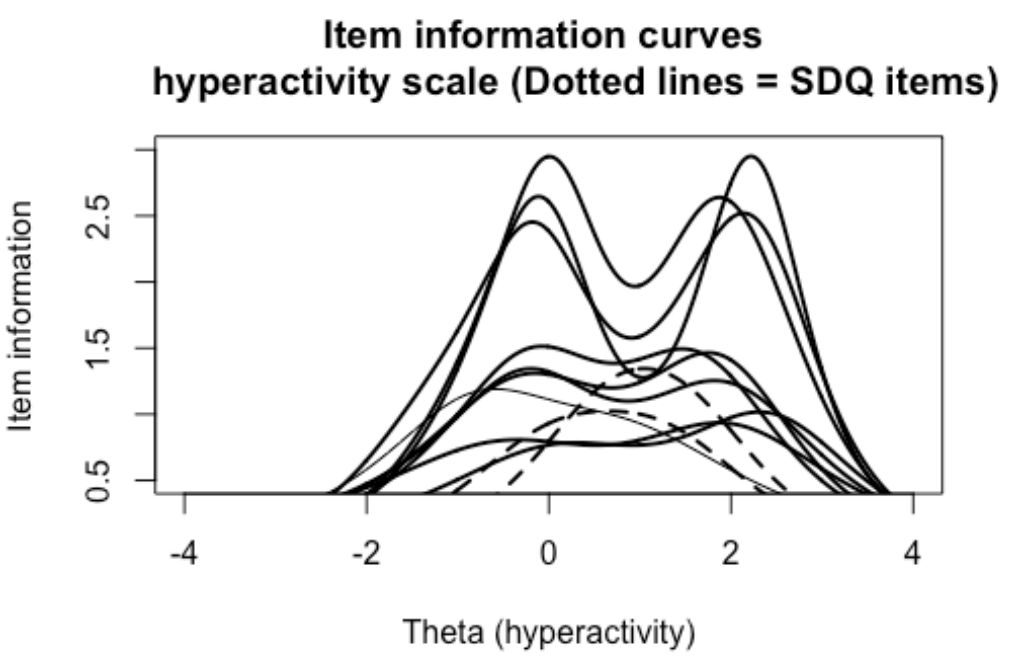

Figure 1: Item information curves of the combined hyperactivity scale that contained 9 SWAN items and $2 \mathrm{SDQ}$ (dotted lines) items. The $\mathrm{Y}$-axis shows the item information and the $\mathrm{X}$-axis the estimated theta value (e.g., degree of hyperactivity). A higher theta value is representative for a higher trait value (e.g., a higher degree of hyperactivity).

An analysis of the item fit of all items based on the results of the GPCM of the combined scale showed that the items that were taken from the SDQ questionnaire ("I am restless" and "I am constantly fidgeting") resulted in a bad fit, suggesting that there were respondents with 
answer patterns that badly predicted by the IRT model. For example, there were more respondents with a certain level of hyperactivity that endorsed category 1 than predicted by the GPCM. Although a pattern like that can also be caused by a general misfit of the IRT model, this issue could not be resolved by choosing another model and was therefore interpreted as misfit of the SDQ items within the combined scale. An analysis that was based on solely the 9 SWAN hyperactivity items showed that this model fitted very well and did not result in any item misfit. Statistics and figures that show item fit for every item based on the 9 SWAN hyperactivity items and the combined scales are not displayed here to save space, but can be obtained from the first author.

\section{Inattention}

The same psychometric analyses were performed for the scale that resulted from combining the inattention items from the SWAN questionnaire (9 items) and the SDQ questionnaire (3 items).

Based on multiple analyses with different IRT models (e.g., partial credit model (PCM), generalized partial credit model (GPCM) and graded response model (GRM)), the GPCM was selected as the best fitting model for the inattention data and used to investigate item information curves of the combined scale as well as item fit of every separate item. The item information curves of all items contained in the combined scale can be found in Figure 2. The item information curves depict the amount of information provided by the items taken from the two

questionnaires (items from the SDQ represented by dotted lines). The $\mathrm{Y}$ axis of the figure shows the item information, plotted against the theta value (X-axis) where a higher value represents a higher degree of inattention. Similar to the hyperactivity scale, the items that were taken from the SDQ questionnaire ("I am easily distracted", "I think before I do things" and "I finish the work I am doing") showed a bad fit. This could not be resolved by applying a different IRT model. A 
GPCM applied to the SWAN items alone resulted in a good fit. Statistics and figures that show item fit for every item based on the 9 SWAN inattention subscale items and the combined scales are not displayed here to save space, but can be obtained from the first author.

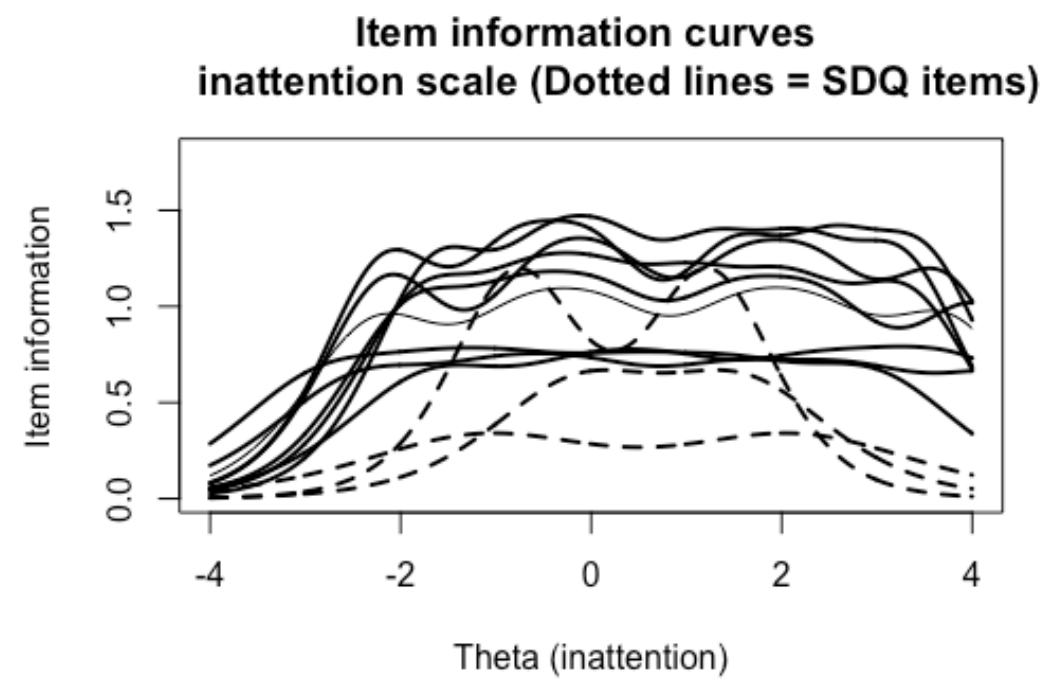

Figure 2: Item information curves of the combined inattention scale that contained 9 SWAN items and 3 SDQ (dotted lines) items. A higher theta value is representative for a higher trait value (e.g., a higher degree of inattention).

\section{Genetic results}

Since the results of the psychometric analyses showed that we cannot combine the two scales, only the items originating from the SWAN questionnaire were used for the genetic analyses. We choose to use the items from the SWAN questionnaire, because 1) the item information curves showed that these items were more informative and 2) the questionnaire contains more items (i.e., 9 hyperactivity items compared to 2 in the SDQ questionnaire and 9 inattention items compared to 3 in the SDQ questionnaire) and will therefore be more reliable. Since the two MCMC chains of the ADE and ADE with GxE model did not achieve stationarity (i.e., did not 
approach the joint posterior or target distribution sufficient closely), the results of these two models are not discussed in the following.

\section{Hyperactivity}

An item-level analysis of all 9 SWAN questionnaire hyperactivity items resulted in a Cronbach's alpha equal to 0.89 with a lowest item-rest correlation equal to 0.43 . The distribution of sumscores (based on all MZ and DZ twins without any missing item scores) can be found in Figure 3. Sum-scores correlated 0.47 among MZ twins and 0.19 among DZ twins.

\section{Distribution of sum-scores}

(SWAN hyperactivity items)

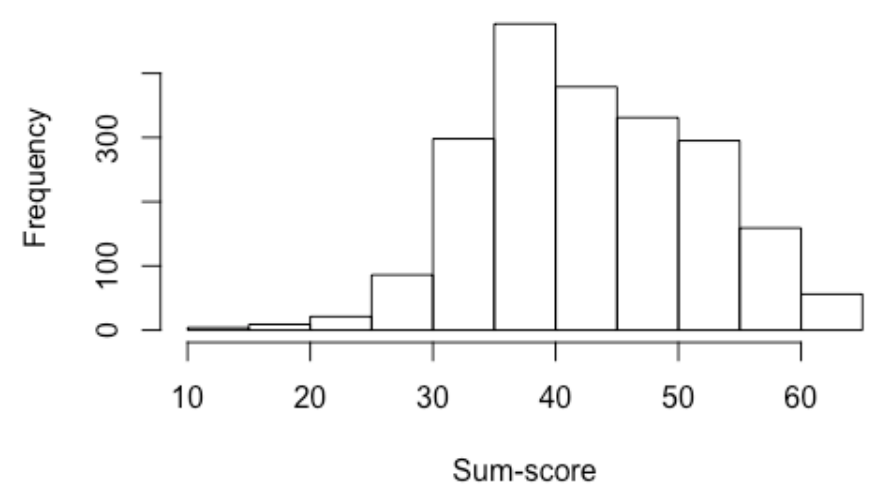

Figure 3. Distribution of the sum-scores of all 9 hyperactivity items (based on all MZ and DZ twins with complete data). A higher sum-score is representative for a higher value on the trait (e.g., a higher degree of hyperactivity).

Table 1 presents the DIC for all fitted genetic models. The AE model with AxE showed the lowest DIC and was therefore chosen as the preferred model for our data. 
Table 1. Hyperactivity: Model fit (DIC) for all genetic models

\begin{tabular}{|c|c|c|}
\hline \multicolumn{2}{|c|}{ Genetic Model } & DIC \\
\hline \multirow[t]{2}{*}{ I. } & $\mathbf{A E}$ & 51755 \\
\hline & With AxE & 51658 \\
\hline \multirow[t]{2}{*}{ II. } & ACE & 51757 \\
\hline & With AxE & 51661 \\
\hline
\end{tabular}

Note. DIC $=$ deviance information criterion

The posterior means and standard deviations of all parameters as well as narrow-sense heritability $\left(h^{2}\right)$ that resulted from the AE with AxE model can be found in Table 2. Narrowsense heritability is the proportion of the total observed variance that can be explained by additive genetic influences and was defined here as $\frac{\sigma_{A}^{2}}{\sigma_{A}^{2}+\exp \left(\beta_{0}\right)}$. Most of the observed variance in hyperactivity could be explained by additive genetic influences, resulting in a narrow-sense heritability equal to $57 \%$. Furthermore, a substantial part of the variance could be explained by unique-environmental influences. Analyses furthermore resulted in a positive and significant AxE interaction, meaning that twins with a high genotypic value showed more variance in hyperactivity due to unique-environmental influences than twins with lower genotypic values. The effect size of this interaction effect was equal to 3.00 which can be regarded as a large effect (see Schwabe \& van den Berg, 2014 for technical details).

Table 2. Hyperactivity: Estimates of all parameters and narrow-sense heritability, based on the AE model with AxE interaction.

Posterior mean (SD) HPD 


\begin{tabular}{ccc}
\hline$\sigma_{A}^{2}$ & $0.25(0.03)$ & {$[0.19 ; 0.31]$} \\
\hline $\exp \left(\beta_{0}\right)$ & $0.19(0.03)$ & {$[0.14 ; 0.24]$} \\
\hline$\beta_{1}(\mathrm{AxE})$ & $2.20(0.22)$ & {$[1.79 ; 2.65]$} \\
\hline$h^{2}$ & $0.57(0.04)$ & {$[0.48 ; 0.65]$}
\end{tabular}

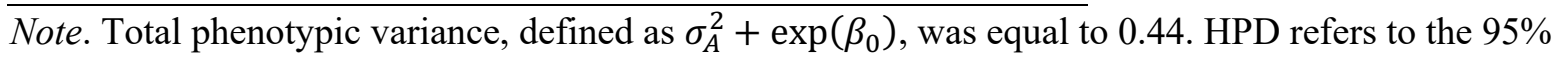
highest posterior density interval and $h^{2}$ to narrow-sense heritability.

The $95 \%$ credibility region of the $\mathrm{AxE}$ interaction effect is displayed for the entire range of estimated genotypic values in Figure 4.

\section{5\% Credibility region $\mathrm{AxE}$ interaction}

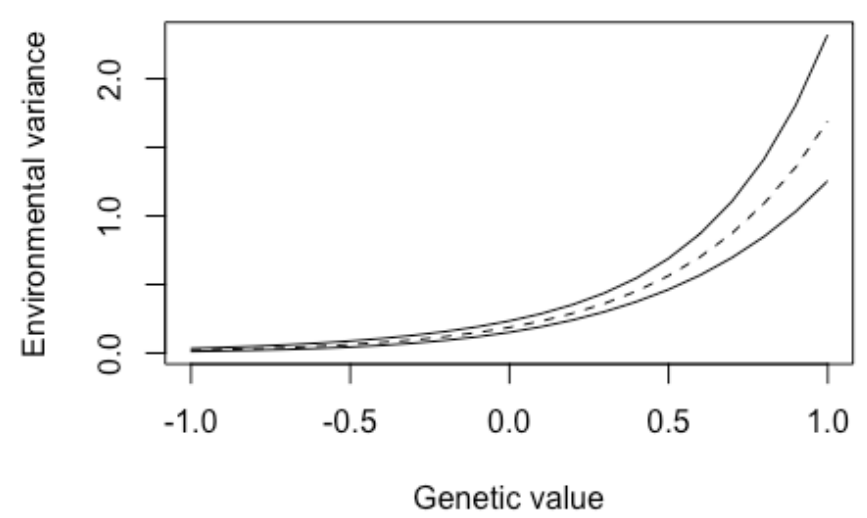

Figure 4. Hyperactivity: $95 \%$ credibility region of the AxE interaction. The entire range of estimated genetic values (i.e., all posterior means of all twins) is displayed on the $X$-axis with the respective (unstandardized) environmental variance, calculated as $\sigma_{E j}^{2}=\exp \left(\beta_{0}+\beta_{1} A_{j}\right)$ on the $Y$-axis. A higher genetic value is representative for a higher genetic predisposition for hyperactivity.

\section{Inattention}

An item-level analysis of all 9 SWAN questionnaire inattention items resulted in a Cronbach's alpha equal to 0.89 with lowest item-rest correlation equal to 0.45 . The distribution of sumscores (based on all MZ and DZ twins without any missing item scores) can be found in Figure 
5. Sum-scores correlated 0.46 among MZ twins and 0.24 among DZ twins.

\section{Distribution of sum-scores}

(SWAN inattention items)

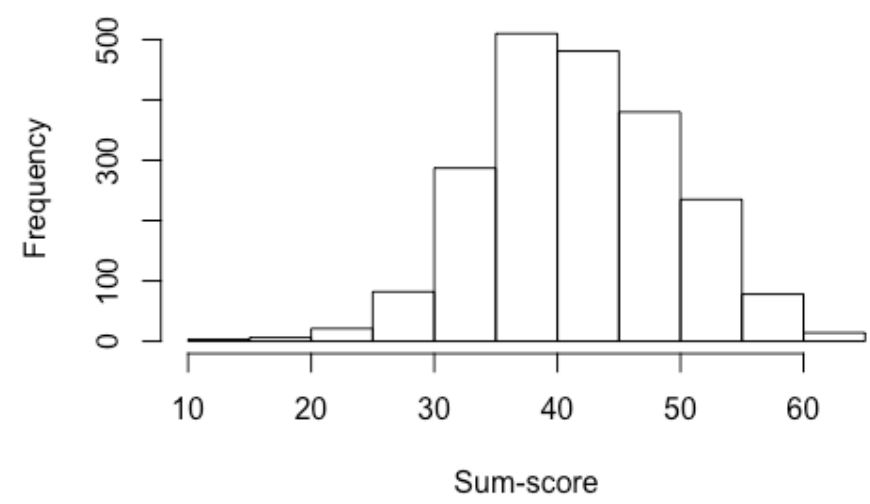

Figure 5. Distribution of the sum-scores of all 9 inattention items (based on all MZ and DZ twins with complete data). A higher sum-score is representative for a higher value on the trait (e.g., a higher degree of inattention).

The DIC for all fitted genetic models can be found in Table 3. The ACE model with AxE showed the lowest DIC and was therefore chosen as the preferred model for the twin data.

Table 3. Model fit (DIC) for all genetic models.

\begin{tabular}{ccc}
\hline \multicolumn{2}{l}{ Genetic Model } & DIC \\
\hline I. & AE & 49919 \\
& With AxE & 49884 \\
& & \\
\hline II. & ACE & 49917
\end{tabular}

With AxE 49857

Note. DIC $=$ deviance information criterion.

Posterior means and standard deviations of all parameters as well as narrow-sense heritability 
based on the ACE with AxE model can be found in Table 4. In contrast to the results of the hyperactivity scale, unique-environmental influences were the most important source to explain individual differences in inattention, with a narrow-sense heritability equal to $24 \%$. Furthermore, a positive and significant $\mathrm{AxE}$ interaction was found, meaning that twins with a high genotypic value showed more variance due to unique-environmental influences than twins with lower genotypic values. The $95 \%$ credibility region of this interaction effect is displayed for the entire range of estimated genotypic values in Figure 6. The effect size of the AxE interaction effect was equal to 3.07 which can be regarded as a large effect (see Schwabe \& van den Berg, 2014 for more technical details).

Table 4. Inattention: Estimates of all parameters and narrow-sense heritability, based on the ACE model with AxE interaction.

\begin{tabular}{ccc}
\hline & Posterior mean (SD) & HPD \\
\hline$\sigma_{A}^{2}$ & $0.27(0.07)$ & {$[0.14 ; 0.41]$} \\
\hline$\sigma_{C}^{2}$ & $0.31(0.05)$ & {$[0.20 ; 0.41]$} \\
\hline $\exp \left(\beta_{0}\right)$ & $0.51(0.07)$ & {$[0.37 ; 0.65]$} \\
\hline$\beta_{1}(\mathrm{AxE})$ & $2.16(0.32)$ & {$[1.56 ; 2.79]$} \\
\hline$h^{2}$ & $0.24(0.06)$ & {$[0.14 ; 0.36]$}
\end{tabular}

Note. Total phenotypic variance, defined as $\sigma_{A}^{2}+\sigma_{C}^{2}+\exp \left(\beta_{0}\right)$, was equal to 1.09 . HPD refers to the $95 \%$ highest posterior density interval and $h^{2}$ to narrow-sense heritability. 


\section{5\% Credibility region AxE interaction}

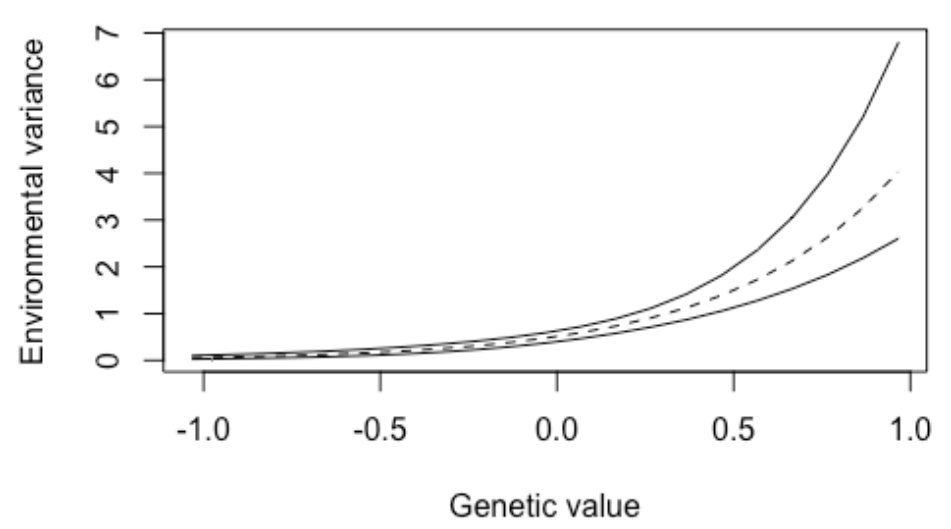

Figure 6. Inattention: $95 \%$ credibility region of the AxE interaction. The entire range of estimated genetic values (i.e., all posterior means of all twins) is displayed on the $X$ axis with the respective (unstandardized) environmental variance, calculated as $\sigma_{E j}^{2}=\exp \left(\beta_{0}+\beta_{1} A_{j}\right)$ on the $Y$ axis. A higher genetic value is representative for a higher genetic predisposition for inattention.

\section{Discussion}

The results of decades of twin studies make it undebatable that ADHD is a heritable disorder. However, there is emerging evidence that genetic- and environmental influences on ADHD interact. Although studies suggest that these interactions are important phenomena in the aetiology of mental diseases in general, research on genotype-environment interaction has not been a focus of genetically-informative ADHD studies. Here, we investigated genotypeenvironment interaction in explaining individual differences in ADHD by analysing the itemlevel scores of 2168 twins who completed two questionnaires that measure the two ADHD core dimensions, hyperactivity and inattention - the Strengths and Difficulties Questionnaire (SDQ) and the Strength and Weaknesses of ADHD Symptoms and Normal Behavior (SWAN) questionnaire. Specifically, it was investigated whether a genetic predisposition for hyperactivity 
(inattention) determines the extent to which unique-environmental influences explain variability in hyperactivity (inattention).

Establishing a measure with good psychometric properties is important for finding genomic signals (see van der Sluis et al., 2010; van den Berg \& Service, 2012 \& Schwabe et al., 2019). To maximize the psychometric information on ADHD symptoms, here, a thorough psychometric evaluation of the questionnaires was performed to investigate whether the items from the two questionnaires could be combined to form two longer subscales. This would improve the reliability of the measures. Multiple analyses suggested however that the items from the different questionnaires do not measure the same latent construct and hence cannot be used in the same analysis to reflect the same construct. This is an important result, since genomic studies often combine data from multiple cohorts to increase power to find genetic signals (van den Berg et al., 2014). Based on the results of this study, we advise researchers to only use ADHD data from individuals that were assessed by the same questionnaire (either SWAN or SDQ).

The psychometric analyses furthermore showed where in the distribution of hyperactivity and inattention scores there is the most phenotypic information. With regard to the hyperactivity subscales, it can be concluded that the subscales provide information mostly on individuals in the middle range of hyperactivity (e.g., individuals who show neither a very low or a very high degree of hyperactivity). On the other hand, the inattention subscales provide considerable information at the middle range and higher end of the spectrum (i.e., individuals with a high degree of inattention). This is an important results since the power to detect a quantitative trait locus (QTL) does not only depend on variation in genotypes and variation in liability, but also on how well the measurement tool that is used to assess liability discriminates among the genotypes. Improving the scale that is used to measure the phenotype might be much cheaper than ever- 
increasing sample sizes with the same clinical test. Using simulation studies, van den Berg \& Service (2012) show that, when looking for alleles of small effect, tests with normally distributed item parameters give the largest power for a wide range of allele frequencies, and for different study designs. Relating this to genome-wide association studies (GWAS) in ADHD research, we advise researchers to carefully investigate test information curves of the used scale for the respective sample of genotyped individuals. Investigating test information curves can aid in identifying possible measurement issues and power can be enhanced by simply adding items to a scale or trivial changes such as rewording an extreme item in case of a ceiling or floor effect. For example, changing "I like chocolate very much" into "I like chocolate" will make the item less extreme and as such increase prevalence of positive responses. This will result in lower item difficulty parameter values and therefore the maximum of the respective information function will be lower on the liability scale, with a corresponding change in statistical power (example adapted from van den Berg \& Service, 2012).

Based on the results of the psychometric analyses, genetically-informative analyses that included genotype-environment interaction were restricted to the items from the SWAN questionnaire. An $\mathrm{AE}$ with $\mathrm{AxE}$ interaction model based on the hyperactivity data resulted in a moderate narrow-sense heritability (57\%), which is comparable to the results of earlier studies (e.g., a meta-analysed narrow-sense heritability equal to $71 \%$, see Nikolas \& Burt, 2010). Furthermore, while the finding that common-environmental influences contributed a negligible part to the observed variance was also in line with earlier research, the model resulted in a slightly higher estimate of unique-environmental influences. Contrary to earlier research, the results of our ACE with AxE model suggest that the most important source of explaining variance in the inattention dimension were unique-environmental influences while both additive 
genetic influences as well as common-environmental influences also contributed a significant part to explaining the observed variance. One reason for this seemingly contradiction could be that the results of earlier research were based on the analysis of possibly skewed sum-scores whereas all models estimated here were based on latent trait scores.

The most important result of this research is that, on both core dimensions of ADHD, we found significant genotype-environment interaction (AxE): the unique-environmental variance component is larger in adolescents with a genetic predisposition towards high hyperactivity (inattention) than in adolescents with a genetic predisposition towards low hyperactivity (inattention). Effect sizes and credibility regions of these interaction effects suggest that these concern large and robust effects. This finding contributes to our understanding of the aetiology of ADHD, since this suggests that adolescents who are genetically predisposed to have a high degree of hyperactivity (inattention) are more sensitive to unique-environmental influences (alternatively, these influences play a larger role). The method that was used in this research to investigate genotype-environment interaction in ADHD was parametrized such that, both genetic as well as unique-environmental influences, were modelled as latent (i.e., unmeasured) variables. Although this results in great statistical power to determine in an exploratory way whether there is genotype-environment interaction at all (see Schwabe \& van den Berg, 2014 for technical details), a drawback is that we do not know what specific unique-environmental measures create more individual differences in adolescents genetically predisposed to a high degree of hyperactivity and inattention. Future research on the aetiology of ADHD should focus on the exact nature of the effect found here by collecting specific environmental measures at the individual level. These should specifically be tested in adolescents with many ADHD symptoms, since that is where these environmental factors operate. Given that there is a broad range of 
influences that can contribute to differences in twin pairs (ranging from subtle prenatal differences to different perceptions of the environment), future research should first focus on variables that have proven to be important for ADHD, like parental stress (Tzang, Chang \& Liu, 2008) and adverse childhood experiences like socioeconomic hardship or neighbourhood violence (Brown et al., 2014).

\section{Acknowledgements}

We gratefully acknowledge the ongoing contribution of the participants in the Twins Early Development Study (TEDS) and their families. TEDS is supported by a program grant to RP from the UK Medical Research Council (MR/M021475/1 and previously G0901245), with additional support from the US National Institutes of Health (AG046938). RP is supported by a Medical Research Council Professorship award (G19/2). KR is supported by a Sir Henry Wellcome Postdoctoral Fellowship. AA and MJ are funded by the European Union's Horizon 2020 research and innovation programme under the Marie Sklodowska-Curie grant agreement no. 721567.

\section{References}

American Psychiatric Association (2008). Diagnostic and statistical manual of mental disorders: DSM-IV-TR, $4^{\text {th }}$ edn. Washington, DC: American Psychiatric Association.

Box, G., \& Tiao, G. (1992). Bayesian inference in statistical analysis. New York: John Wiley \& Sons. 
Brown, N.M., Brown, S.N., Briggs, R.D., Germán, M., Belamarich, P.F. \& Oyeku, S.O. (2014). Associations between adverse childhood experiences and ADHD diagnosis and severity. Academic Pediatrics, 16 (4), 349-355

Cameron, N.D. (1993). Methodologies for estimation of genotype with environment interaction. Livestock Production Science, 35(3-4), 237-249. DOI: 10.1016/0301-6226(93)90095-Y

Chalmers, R.P. (2012). Mirt: A Multidimensional Item Response Theory Package for the R Environment. Journal of Statisitcal Software, 48 (6), 1-29. Retrieved from https://ideas.repec.org/a/jss/jstsof/v048i06.html

Coghill, D., Soutullo, C., d'Aubuisson, C., Preuss, U., Lindback, T., Silverberg, M., \& Buitelaar, J. (2008). Impact of attention-deficit/hyperactivity disorder on the patient and family: results from a European survey. Child and Adolescent Psychiatry and Mental Health, 2(1), 31. DOI: $10.1186 / 1753-2000-2-31$.

Eaves, L. J., Last, K., Martin, N. G., \& Jinks, J. L. (1977). A progressive approach to non-additivity and genotype-environmental covariance in the analysis of human differences. British Journal of Mathematical and Statistical Psychology, 30(1), 1-42. DOI: 10.1111/j.20448317.1977.tb00722.x

Faraone, S. \& Doyle, A. (2002). The nature \& heritability of attention deficit hyperactivity disorder. Child and Adolescent Psychiatric Clinics of North America, 10, 299-316. DOI: $10.1177 / 070674370200601 \mathrm{~S} 03$

Gelfand, A., \& Smith, A. (1990). Sampling-based approaches to calculating marginal densities. Journal of the American Statistical Association, 85(410), 398-409. DOI: $10.1080 / 01621459.1990 .10476213$ 
Geman, S., \& Geman, D. (1984). Stochastic relaxation, gibbs distributions and the Bayesian restoration of images. IEEE Transactions on Pattern Analysis and Machine Intelligence, 6(6), 721-741. DOI: 10.1109/TPAMI.1984.4767596

Goodman, R. (1997). The Strengths and Difficulties Questionnaire: A Research Note. Journal of Child Psychology and Psychiatry, 38, 581-586

Gould, K. L., Coventry, W. L., Olson, R. K., \& Byrne, B. (2018). Gene-environment interactions in ADHD: the roles of SES and chaos. Journal of abnormal child psychology, 46(2), 251263. DOI: $10.1007 / \mathrm{s} 10802-017-0268-7$

Hanscombe, K.B., Trzaskowski, M., Haworth, C.M.A., Davis, O.S.P., Dale, P.S., Plomin, R. (2012). Socioeconomic status (SES) and children's intelligence (IQ): in a UKrepresentative sample SES moderates the environmental, not genetic, effect on IQ. PlOsOne, 7(2):e30320

Hudziak, J.J., Rudiger, L.P., Neale, M.C., Heath, A.C. \& Todd, R.D. (2000). A twin study of inattentive, aggressive, and anxious/depressed behaviors. Journal of the American Academy of Child and Adolescent Psychiatry, 39, 469-476. DOI: 10.1097/00004583200004000-00016

Martin, N. (2000). Gene-environment interaction and twin studies. Advances in twin and sib-pair analysis. London: Greenwich Medical Media Ltd, 143-150. Retrieved from https://keppel.qimr.edu.au/contents/publications/staff/CV252_OCR.pdf

Molenaar, D., \& Dolan, C. V. (2014). Testing systematic genotype by environment interactions using item level data. Behavior genetics, 44(3), 212-231. DOI: 10.1007/s10519-014-96479 
Muraki, E. (1992). A Generalized Partial Credit Model: Application of an EM Algorithm. Applied Psychological Measurement, 16(2), 159-176. DOI: 10.1177/014662169201600206

Nikolas, M.A. \& Burt, S.A. (2010). Genetic and Environmental Influences on ADHD Symptom Dimensions of Inattention and Hyperactivity: A Meta-Analysis. Journal of Abnormal Psychology, 119(1), 1-17. DOI: 10.1037/a0018010

Plummer, M. (2003). JAGS: A program for analysis of Bayesian graphical models using Gibbs sampling. In Proceedings of the 3rd international workshop on distributed statistical $\begin{array}{llll}\text { computing } & 124(125.10), & 1-10 . & \text { Retrieved }\end{array}$ http://www.ci.tuwien.ac.at/Conferences/DSC-2003/Drafts/Plummer.pdf

Plummer, M. (2013). rjags: Bayesian graphical models using mcmc [Computer software manual]. Retrieved from http://CRAN.R-project.org/package=jags (R package version 3 10)

Polanczyk, G., De Lima, M. S., Horta, B. L., Biederman, J., \& Rohde, L. A. (2007). The worldwide prevalence of ADHD: a systematic review and metaregression analysis. American journal of psychiatry, 164(6), 942-948. DOI: 10.1176/ajp.2007.164.6.942

R Development Core Team. (2008). R: A language and environment for statistical computing [Computer software manual]. Vienna, Austria. Retrieved from http://www.R-project.org (ISBN 3-900051-07-0)

Rietveld, M.J., Hudziak, J.J., Bartels, M., van Beijsterveldt, C.E. \& Boomsma, D.I. (2004). Heritability of attention problems in children: Longitudinal results from a study of twins, age 3 to 12. Journal of Child Psychology and Psychiatry, 45, 577-588. DOI: $10.1111 / \mathrm{j} .1469-7610.2004 .00247 . x$

Rimfeld, K., Malanchini, M., Spargo, T., Spickernell, G., Selzam, S., McMilan, A., Dale, P.S., Eley, T.C. \& Plomin, R. (2019). Twins Early Development Study: A Genetically Sensitive 
Investigation into Behavioral and Cognitive Development from Infancy to Emerging Adulthood. Twin Research and Human Genetics, 22 (6), 508-513

Rosseel, Y. (2012). lavaan: An R Package for Structural Equation Modeling. Journal of Statistical Software, 48(2), 1-36. Retrieved from

https://www.jstatsoft.org/htaccess.php?volume=48\&type=i\&issue=02\&paper=true

SanCristobal-Gaudy, M., Elsen, J. M., Bodin, L., \& Chevalet, C. (1998). Prediction of the response to a selection for canalisation of a continuous trait in animal breeding. Genetics Selection Evolution, 30(5), 423-451.

Retrieved

from https://gsejournal.biomedcentral.com/track/pdf/10.1186/1297-9686-30-5-423

Scheres, A., Oosterlaan, J., Swanson, J., Morein-Zamir, S., Meiran, N., Schut, H., ... \& Sergeant, J. A. (2003). The effect of methylphenidate on three forms of response inhibition in boys with AD/HD. Journal of abnormal child psychology,31(1), 105-120. DOI: 10.1023/A:1021729501230.

Schmitz, M., Polanczyk, G. \& Rohde, L.A. (2007). ADHD: remission in adolescence and predictors of persistence into adulthood. Jornal Brasileiro de Psiquiatria, 56(1), 25-29.

Schwabe, I. (2017). BayesTwin: An R Package for Bayesian Inference of Item-Level Twin Data. Journal of Open Research Software, 5 (1). doi: 10.5334/jors.185

Schwabe, I., Boomsma, D. I., \& van den Berg, S. M. (2017). Increased environmental sensitivity in high mathematics performance. Learning and individual differences, 54, 196-201. DOI: 10.1016/j.lindif.2017.01.018

Schwabe, I., Jonker, W., \& Van Den Berg, S. M. (2015). Genes, Culture and Conservatism-A Psychometric-Genetic Approach. Behavior genetics, 46(4), 516-528. DOI: 10.1007/s 10519-015-9768-9 
Schwabe, I., Milaneschi, Y., Gerring, Z., Sullivan, P. F., Schulte, E., Suppli, N. P., ... \& Middeldorp, C. M. (2019). Unraveling the genetic architecture of major depressive disorder: merits and pitfalls of the approaches used in genome-wide association studies. Psychological Medicine, 49(16), 2646-2656. DOI: 10.1017/S0033291719002502

Schwabe, I., \& van den Berg, S.M. (2014). Assessing genotype by environment interaction in case of heterogeneous measurement error. Behavior Genetics, 44(4), 394-406. DOI: $10.1007 / \mathrm{s} 10519-014-9649-7$

Spiegelhalter, D., Best, N., Carlin, B., \& van der Linde, A. (2002). Bayesian measures of model complexity and fit. Journal of the Royal Statistical Society, 64(4), 583-639. DOI: $10.1111 / 1467-9868.00353$

Swanson, J., Schuck, S., Mann, M., Carlson, C., Hartman, K., Sergeant, J., Clevenger, W., Wasdell, M., McCleary, R. (2006). Categorical and dimensional definitions and evaluations of symptoms of ADHD: the SNAP and SWAN rating scales. Irvine: University of California

Tzang, R., Chang, Y. \& Liu, S. (2008). The association between children's ADHD subtype and parenting stress and parental symptoms. International Journal of Psychiatry in Clinical Practice, 13 (4), 318-325

van den Berg, S. M., Beem, L., \& Boomsma, D. I. (2006). Fitting genetic models using Markov Chain Monte Carlo algorithms with BUGS. Twin Research and Human Genetics, 9(3), 334-342. DOI: 10.1375/twin.9.3.334

van den Berg, S. M., Glas, C. A., \& Boomsma, D. I. (2007). Variance decomposition using an IRT measurement model. Behavior genetics, 37(4), 604-616. DOI: 10.1007/s10519-007-91561 
van den Berg, S. M., \& Service, S. K. (2012). Power of IRT in GWAS: Successful QTL mapping of sum score phenotypes depends on interplay between risk allele frequency, variance explained by the risk allele, and test characteristics. Genetic epidemiology, 36(8), 882-889. DOI: $10.1002 /$ gepi.21680

Van Der Sluis, S., Dolan, C. V., Neale, M. C., Boomsma, D. I., \& Posthuma, D. (2006). Detecting genotype-environment interaction in monozygotic twin data: comparing the Jinks and Fulker test and a new test based on marginal maximum likelihood estimation. Twin Research and Human Genetics, 9(3), 377-392. DOI: 10.1375/twin.9.3.377

Van Der Sluis, S., Verhage, M., Posthuma, D., \& Dolan, C. V. (2010). Phenotypic complexity, measurement bias, and poor phenotypic resolution contribute to the missing heritability problem in genetic association studies. PLoS One, 5(11), e13929. DOI: 10.1371/journal.pone.0013929

Wermter, A. K., Laucht, M., Schimmelmann, B. G., Banaschweski, T., Sonuga-Barke, E. J., Rietschel, M., \& Becker, K. (2010). From nature versus nurture, via nature and nurture, to gene $\times$ environment interaction in mental disorders. European child \& adolescent psychiatry, 19(3), 199- 210. DOI: 10.1007/s00787-009-0082-z

Willcutt, E. (2005). The etiology of ADHD: Behavioral and molecular genetic approaches. Cognitive and affective neuroscience of psychopathology. Oxford: Oxford University Press.

\section{Appendix:}

The Inattention \& Hyperactivity subscales of the SWAN (Swanson, 2015) and SDQ 
questionnaires (Goodman, 1997). The items were part of the test booklet that was sent to twins at the age of 16 and answered by themselves.

\section{SWAN}

Inattention

1. Give close attention to detail and avoid careless mistakes

2. Sustain attention on tasks or play activities

3. Listen when spoken to directly

4. Follow through on instructions and finish school work or chores

5. Organise tasks and activities

6. Engage in tasks that require sustained mental effort

7. Keep track of things necessary for activities

8. Ignore extraneous stimuli

9. Remember daily activities

Hyperactive/Impulsive

1. Sit still (control movement of hands or feet or control squirming)

2. Stay seated (when required by class rules or social conventions)

3. Modulate motor activity (inhibit inappropriate running or climbing)

4. Play quietly (keep noise level reasonable)

5. Settle down and rest (control constant activity)

6. Modulate verbal activity (control excessive talking)

7. Reflect on questions (control blurting out answers)

8. Await turn (stand in line and take turns)

9. Enter into conversations \& games without interrupting or intruding

\section{SDQ}

Hyperactive/Inattention

1. I am restless

2. I am constantly fidgeting

3. I am easily distracted

4. I think before I do things

5. I finish the work I am doing 